\title{
The Relative Importance of Health Care and Social Services for Population Health: A Time Series Investigation
}

\author{
Benjamin Yawney ${ }^{1} \&$ Akhter Faroque ${ }^{1}$ \\ 1 Department of Economics, Laurentian University, Ontario, Canada \\ Correspondence: Akhter Faroque, Department of Economics, Laurentian University, Ramsey Lake Road, \\ Sudbury, Ontario, P3E 2C6, Canada. E-mail: afaroque @laurentian.ca
}

Received: July 30, 2019

Accepted: August 16, 2019

Online Published: September 25, 2019

doi:10.5539/ijef.v11n10p93

URL: https://doi.org/10.5539/ijef.v11n10p93

\begin{abstract}
We study the relative importance of government health care and social services spending in the short, medium and long run across vector error correction models for six population health indicators. Each model takes into account the key time series properties of the health input and output data and also controls for the broader socio-economic, demographic, life-style and environmental determinants of health. The evidence shows that both types of spending contribute significantly to extending life expectancy and lowering mortality. However, the relative contributions of health care spending are bigger in the short run, while those of social services spending are bigger in the medium and the long run. Any policy of re-allocation of resources from health care to social services must take this trade-off into account.
\end{abstract}

Keywords: health care, social services, vector error correction model

\section{Introduction}

During the past several decades, rich countries have persistently invested an increasing share of their growing GDP on health care, much of it through their public health care systems. The vast majority of these expenditures were made for sickness care, particularly for hospital care, physician services and drugs. In more recent years, concern about the sustainability of escalating health care costs (Skinner et al., 2011; Liaropolos \& Goranitis, 2015) combined with growing evidence that government spending on social services are strongly associated with better health outcomes (McDaid et al., 2015), has stimulated a fascinating new line of enquiry.

The new line of research addresses a fundamental question: whether secular growth in healthcare spending may have exhausted most of the easy medical interventions to extend life and reduce death, thereby severely depressing the marginal health impact of health care spending. Already, evidence from several studies has shown that the ratio of social services to health care spending is associated with higher life expectancy at birth and lower mortality rates (Bradley et al., 2011; Bradley et al., 2016; Dalton et al., 2018). This strand of the literature has, therefore, recommended that a policy of shifting the composition of government budgets more towards social services and away from health care may achieve better health outcomes, without requiring an increase the size of the budget.

We take the findings of the existing literature noted above as our point of departure and extend the analysis of the health effects of government spending on health and social services in two important directions. First, we explore the relative health effects of these two public sector determinants of health in the short, medium, and the long run. Second, the model and methodology we use to isolate the marginal health effects of spending on health and social services is markedly different from those typically used in previous studies. Most existing studies rely on classical regression models augmented with deterministic time trends to achieve the separation; by contrast, the vector error correction (VEC) models we use are largely dictated by the data properties of the observational data available to us (Canadian national data: 1980-2014). Our goal in making these extensions is to see if the traditional policy prescription of a reallocation of resources from health care to social services holds up in these broader contexts.

The VEC models we estimate have several advantages over the classical lineal regression models typically estimated in previous studies. First, the most dominant component of health data is the long-run trend component and the VEC models account for the trend, not simply as a predictable time trend, rather by modelling it as 
long-run equilibrium relationship between a health indicator and the health determinants that are cointegrated (jointly stationary) with it. Second, simultaneity and reverse causality which have often been a potential source of estimation biases in traditional studies is not a concern with VEC models, because these models treat all variables as endogenous. Finally, the VEC models allow us to draw inferences about the relative health effects of government spending on health and social services based not just on statistical significance as in the traditional literature. Rather, we are able to decompose the forecast error variance of each health indicator into (quantitative) proportions that are accounted for by the shocks associated with the indicator itself and also those associated with each of the determinants of health, in the short ( 1 year), medium (5 years) and long (20 years) run.

\section{Methodology}

\subsection{Research Design}

We study the long-run relationships between six different population health indicators and their determinants using vector error correction (VEC) models and a national time series dataset for Canada for the period 1980-2014. All data are publicly available and are drawn from three sources: Statistics Canada (CANSIM), Canadian Institute of Health Information (CIHI) and the data appendix of Kneebone and Watkins (2016); the last of these is our source for government social services spending.

Why VEC models? There are two broad approaches to empirical modelling of population health production function. The first approach estimates a theoretical health production function (Grossman, 1972) specified/selected by the authors; most of the traditional empirical studies of population health falls in this category (Bradley et al., 2011; Dalton et al., 2018). An alternative approach allows data properties to determine the choice of models. The empirical VEC models we estimate fall in this latter category. Under this data-driven modelling strategy, the health models are expected to fit the data better and produce more accurate health estimates of the individual determinants of health. However, to achieve such estimation accuracy, it is necessary to guard against potential estimation biases that can arise from three major sources of uncertainty surrounding (i) the choice of variables for the health production function (ii) the unknown properties of the observational data and (iii) the choice of model used to disentangle the marginal health effects of interest. Since each of these sources of uncertainty/challenges can infect and bias the estimates of interest to us, we describe the nature of these uncertainties and how we address them in this paper in sub-sections 2.2, 2.3, 2.4 and 2.5 below.

\subsection{Health Indicators}

National statistical agencies across countries typically report measures of population health by a common and consistent set of health outcome variables such as life expectancy at birth and at other ages, the rates of preventable deaths and child mortality. We include six such health indicators in the present study: life expectancy at birth (for male and female); life expectancy at age 65 (for male and female); premature death and infant mortality.

\subsection{Determinants of Health: Literature Review}

There is no general consensus about the determinants of health; only an agreement that health is determined by the interaction of many different factors. We address this uncertainty here by conducting a brief survey of the relevant literature, with the aim to assemble a set of variables that are most likely to have a positive or negative effect on the health indicators. We describe these factors below under five sub-headings: the public-sector determinants; the socio-economic, demographic, life-style and the environmental determinants of health.

\subsubsection{Public-Sector Determinants of Health}

The health effects of the two public sector determinants of population health which are of primary interest to this paper are government spending on health care and on social services in Canada. Over the past several decades government spending on health care across Canadian provinces has growth rapidly, the vast majority of the spending being made on hospital care, physician services and drugs, after a person falls sick. Expenditures on such sickness care are expected to have an immediate and direct effect on population health indicators. Over the same period, provinces have also invested on a host of community-based social services including affordable housing; nutritional support for women, infants, children and adults and outreach programs. While these services may or may not have a direct effect on health, they may be viewed as a form of preventive health spending that reduces the health risks to the broader population, rather than treating those with disease (McDaid et al., 2015). Such services are expected to have beneficial longer term effect on the health indicators.

Somewhat surprisingly, a considerable body of cross-country empirical evidence shows little correlation between health care spending and health outcomes (Hitris \& Posnet, 1992; Wilkinson \& Reich, 2011). One possible reason for this is diminishing marginal returns to health care spending. However, the evidence from Canadian 
studies is more favourable, as they generally show that health care spending extends life expectancy and prevents death (Cremieux et al., 1999; 2000). The few statistical investigations that have been undertaken in recent years all show that social services spending have significant health benefits (Bradley et al., 2016; Dalton et al., 2018; Dahlgren \& Whitehead, 1991).

\subsubsection{Socio-Economic Determinants of Health}

Besides the public sector determinants of health, we also include three broader socio-economics variables as potential determinants of health. Since Canada's public (tax-financed) health system covers only about 70 percent of annual health care spending per capita (the rest must be financed with out-of-pocket funds), we include a measure of household income and two proxies for inequality in the distribution of income (poverty rate (Note 1) and the unemployment rate). A person's income is expected to be beneficial to health, given that health care services are generally regarded as normal goods. By the same token, income inequality is expected to have negative effects on health outcomes because people below a certain minimum threshold of income may not be able or willing to shift expenditure from consumption to health care to extend life, since their marginal utility of consumption remains high. Besides the direct effect of income inequality on health via consumption, inequality may also tear into 'human psyche, creating anxiety, distrust and an array of mental and physical ailments' (Wilkinson \& Pickett, 2011).

\subsubsection{Demographic Determinants}

Following previous studies (Cremieux et al., 1999; Dutton et al., 2018), we include age distribution (denoted by the proportion of population who are sixty-five years of age or older) and also gender (denoted by the proportion of female in the population) as potential demographic determinants of health in our empirical health models. The age distribution of the population and how it changes over time may have an important effect on health outcomes in the long run. For example, a region/province of the country with a higher proportion of elderly people will require more health care spending to produce similar health outcomes compared to another province with a lower proportion of elderly people. In addition, gender can be a determinant of health, since men and women tend to suffer from different types of diseases and also exhibit quite different health outcomes.

\subsubsection{Life-Style Determinants}

It is long recognized that the health status of individuals depends on individual lifestyle choices, especially, with regard to smoking, drinking and nutrition intake. Evidence shows that smoking (nicotine intake) damages the lungs, heart and blood circulation, increasing the long-term risk of coronary disease, heart attack, stroke, cancer, and damaged arteries that supply blood to the brain (WHO, 2001). Heavy drinking can be traced to liver disease, pancreatitis, ulcers, immune system dysfunction, osteoporosis and brain damage. These debilitating health conditions may significantly reduce life expectancy and increase preventable deaths in the population. We include expenditure on smoking and drinking as separate control variables in our empirical health models.

\subsubsection{Urbanization and Environmental Determinants}

Urbanization and environmental factors can also have a lasting effect on population health. The health effects of urbanization are complex because some aspects of urbanization can be beneficial to health, while others are certainly detrimental to health. For example, 'healthy urbanization' which provides improved access to medical care, better housing and better jobs can lead to higher life expectancy and lower mortality in the long run. But unplanned and unhealthy urbanization which results in the growth of city slums and stark income inequality may make larger segments of the population vulnerable to type II diabetes, cardiovascular diseases and obesity (WHO, 1991; Phillips, 1993). So, the impact of urbanization on health outcomes can be positive or negative in any particular application. In the present study, we examine the effects of urbanization on the health of Canadians, with urbanization being measured by the proportion of the population that lives in urban areas, as defined by Canada's national statistical agency, CANSIM.

Based on the background literature reviewed above, we have assembled time series data on six health indicators and ten potential determinants of health. The definitions of these variables and the sources of raw data are presented in the data appendix at the end of the paper. It is notable that Statistics Canada does not publish time series data on social services expenditure. We obtained an aggregate series for Canada by summing the ten provincial time series on social services (SS) expenditure provided in the data appendix of Kneebone and Wilkins (2016). We have converted all nominal variables, such as expenditure on health care, social services, tobacco, and alcohol into real, per-capita terms, by delating each series by the CPI (2002) and by the population series. Finally, we have taken the natural logarithm of all sixteen heath indicators and their potential determinants, before studying data properties. 


\subsection{Data Properties}

A key assumption of all time-series regression models is that the variables included in the models are stationary. A time series is stationary if the mean, variance and the auto-covariance structure of the series do not change with time. In practice, however, time series data in economics typically contain changing mean (trend) and/or changing variance (heteroscedasticity). It is common practice to apply the log transformation to stabilize the variance of a time series; but the treatment of the trend is more controversial. This is because in some cases changes in mean of the series are perfectly predictable, such as a deterministic time trend. But in other cases, period-to-period changes in the mean of the series may be stochastic (unpredictable). Thus, it is important to determine the nature of the trend (deterministic or stochastic) for purposes of modelling time series data.

The most common source of stochastic trend is a unit root (such as in a random walk model). Accordingly, we apply a battery of unit root (ADF, PP and KPSS) tests to each health indicator and their determinants. The null hypothesis for the ADF and the PP tests is that the series has a unit root (stochastic trend), and the alternative is that the series is stationary around a time trend. The null hypothesis for the KPSS test is that the series is stationary around a time trend and the alternative is that it has a unit root (stochastic trend). Since the ADF and PP tests tend to over reject the null hypothesis, while the KPSS test tend to under reject the null, applying both tests allows us to draw robust inferences about the nature of the long term trend contained in each series. For example, if the ADF and PP tests fail to reject the null and the KPSS rejects the null, then such agreement between the tests provides strong/robust evidence that the series under investigation contains a stochastic trend. In case of a conflict between the tests, we can only draw weak inferences about the nature of the long term trend.

Table 1 provides a brief description of each series, along with the notation we use to denote the series. Table 1 also reports the p-values for the ADF and PP unit root tests, and the estimated KPSS test statistic. The evidence shows that, for four of the six health indicators, there is robust support across all three tests for the existence of a unit root (stochastic trend); for the remaining two health indicators there is weak support (not all three tests agree) for the existence of a unit root. The evidence also shows that for six of the ten determinants of health, there is uniform support across all tests for the existence of a stochastic trend; for the remaining four series there is weak support for the existence of a unit root. For none of the series is there uniform support across all tests for a deterministic time trend.

Table 1. The augmented Dickey-Fuller (ADF) and Phillip-Perron (PP) unit root tests and the Kwiatkowski-Phillip-Schimdt-Shin (KPSS) stationary test results: Canadian national health data 1980-2014

\begin{tabular}{lllll}
\hline Variable Description & (Notation) & ADF & PP & \multicolumn{1}{l}{ KPSS } \\
P value & P value & Stat. \\
\hline Health Indicators & & & & \\
Infant Mortality; Per 1000 Live Births & & $.032^{*}$ & $.005^{*}$ & $.212^{*}$ \\
Pre-mature Death; Per 100,000 & LN(IM) & .995 & .995 & $.166^{*}$ \\
Life Expectancy at Birth - Female; Years & LN(PD) & .305 & .510 & .114 \\
Life Expectancy at Birth - Male; Years & LN(LE0F) & .498 & .887 & .105 \\
Life Expectancy at 65 - Female; Years & LN(LE0M) & .645 & .935 & $.157^{*}$ \\
Life Expectancy at 65 - Male; Years & LN(LE65F) (Note 2) & .416 & .516 & $.199^{*}$ \\
Determinants of Health & LN(LE65M) & & & \\
Real Healthcare Expenditure Per Capita & & .418 & .781 & $.147^{*}$ \\
Real Social Services Spending Per Capita & LN(HE) & .358 & .680 & $.146^{*}$ \\
Real Household Gross Domestic Income Per Capita & LN(SS) & .906 & .872 & $.180^{*}$ \\
Real Household Spending on Tobacco Products & LN(GDI) & .259 & .731 & .110 \\
Real Household Spending on Alcohol & LN(TB) & .937 & .937 & $.176^{*}$ \\
Poverty Rate & LN(ALC) & .22 & .218 & .116 \\
Proportion of Females in Population & LN(PR) & .532 & .462 & $.208^{*}$ \\
Proportion of Population Living in Urban Areas & LN(SEX) & .14 & .724 & .101 \\
Proportion of population over 65 years of age & LN(URB) & 1.00 & 1.00 & .142 \\
Unemployment Rate & LN(PP65) & $.001^{*}$ & .116 & .085 \\
\hline
\end{tabular}

Note. Critical values for the KPSS test at the $1 \%, 5 \%$ and $10 \%$ significance levels are $0.216,0.146$ and 0.119 respectively. * represents significance at the $5 \%$ level.

The evidence presented in Table 1 raises an important question: Are the stochastic trends observed in the health indicators the result of similar trends in the determinants of health or are they independent of each other? If, for 
example, the trends in the health indicators are imparted by secular cumulative growth in health care spending or social services spending, then retaining this long-term information is important for modelling the health production function. Accordingly, we formally test to see if a health indicator is jointly stationary with the determinants of health, by applying Johansen's (1995) multivariate cointegration tests. To facilitate drawing robust inferences, we look only for combinations of the determinants of health that are jointly cointegrated with each health indicator according to both the multivariate Trace test and the L-Max test.

Table 2 reports the cointegration test results. The leftmost column of table 2 shows each health indicator as well as the determinant of health that are jointly cointegrated with it according to both the Trace and the L-Max tests.

Table 2. Johansen's Multivariate Cointegration Tests: Canada 1980-2014

\begin{tabular}{lccc}
\hline Variables & VAR & Trace Statistic & Max Eigen Statistic \\
& Lags & & \\
\hline LN(LE0F), LN(HE), LN(SS), LN(ALC), LN(PR) & 1 & $90.71^{*}$ & $36.56^{*}$ \\
& & $(69.82)$ & $(33.88)$ \\
LN(LE0M), LN(HE), LN(SS), LN(PR), LN(GDP) & 1 & $109.44^{*}$ & $52.70^{*}$ \\
& & $(69.82)$ & $(33.88)$ \\
LN(LE65F),LN(HE), LN(SS), LN(ALC), LN(TOB), LN(GDP) & 1 & $148.31^{*}$ & $51.43^{*}$ \\
& & $(95.75)$ & $(40.08)$ \\
LN(LE65M), LN(HE), LN(SS), LN(ALC), LN(TOB), LN(SEX) & 1 & $172.18^{*}$ & $66.97^{*}$ \\
LN(PD), LN(HE), LN(SS), LN(ALC), LN(PR) & & $(95.75)$ & $(40.08)$ \\
& 1 & $98.04^{*}$ & 32.58 \\
LN(IM), LN(HE), LN(SS), LN(ALC), LN(PR) & & $(69.82)$ & $(33.88)$ \\
& & $142.50^{*}$ & $62.84^{*}$ \\
\hline
\end{tabular}

Note 1. The Trace and the Max Eigen statistics are alternative tests of the null hypothesis that the variables listed on the left-most cell are not cointegrated. The critical values for the tests at the $5 \%$ significance level are shown in parentheses below the test statistics. * denotes rejection of the null hypothesis at the 0.05 level.

Note 2. In cases where the tests show the presence of more than one cointegrating vectors, we focus on only the relationship that treats a health output indicator (our target variable of interest) as the dependent variable.

The second column of table 2 shows the number of lags in the vector auto-regression (VAR) model specification used to conduct the tests. The remaining two columns report the estimated Trace and the Max Eigen value statistics, each testing the null hypothesis that the group of variables shown in the left-most column are not jointly cointegrated (stationary).

It is evident from table 2 that the log level of each health indicator is cointegrated with the log levels of a sub-set of the determinants of health. This means that first differencing the variables would result in the loss of valuable long run information. Accordingly, following Banerjee et al. (1993) we estimate a separate vector error correction (VEC) model, in log level of the variables, for each health indicator using the maximum likelihood estimator.

\subsection{VEC Models: Specification}

In this section we use the information from column 1 of Table 2 to specify a benchmark VEC model for each health indicator and also report the estimation results. For purposes of illustration and also to facilitate interpretation of the estimation results, here we specify the VEC model only for life expectancy at birth for female (LEOF) corresponding to row 1 of Table 2:

$$
\begin{gathered}
\Delta L N(L E O F)_{t}=c_{0}+\alpha\left\{\mathrm{LN}(L E 0 F)_{t-1}-b_{1} L N(H E)_{t-1}-b_{2} L N(S S)_{t-1}-b_{3} L N(A L C)_{t-1}-b_{4} L N(P R)_{t-1}\right\}+ \\
\left.\left.\left.\left.\lambda_{1}(\Delta \operatorname{LN}(L E 0 F))_{t-1}+\lambda_{2}(\Delta \operatorname{LN}(H E))_{t-1}\right)+\lambda_{3}(\Delta \mathrm{LN}(\mathrm{SS}))_{t-1}\right)+\lambda_{4}(\Delta \mathrm{LN}(\mathrm{ALC}))_{t-1}\right)+\lambda_{5}(\Delta \mathrm{LN}(\mathrm{PR}))_{t-1}\right)+\varepsilon_{t}
\end{gathered}
$$

where $\Delta$ denotes the first-difference operator and the lower case for a variable, say, $\left(\mathrm{x}_{\mathrm{t}}\right)$ denotes the natural log of the variable, $\operatorname{LN}\left(\mathrm{X}_{\mathrm{t}}\right)$. So, the left hand side of equation (1) denotes the natural log of life expectancy at birth for female (LEOF). The expression in braces \{ \} on the right hand side represents the long run equilibrium relationship between $\log$ of $\mathrm{LEOF}$ and the $\log$ of the determinants of health that are cointegrated with it. Note that the cointegration relationship is written in error correction form, so that in long run equilibrium the expression in braces is zero. But, if le0f and its determinants deviate from the long-run relationship, then the error correction 
term (the expression in braces) will be non-zero and either le0f or its determinants will adjust to restore the long-run relationship among the variables. The coefficient $\alpha$ measures the speed of adjustment of the target variable, $\mathrm{LN}(\mathrm{LE} 0 \mathrm{~F})$, towards the long run equilibrium relationship. The $\mathrm{b}_{\mathrm{i}}$ coefficients are long-run elasticities of le0f with respect to each of its determinants. The remaining terms in equation (1) denote 1-period lag of the first difference of each variable in the model; together they capture the short term dynamics between LN(LE0F) and its determinants. Finally, $\varepsilon_{\mathrm{t}}$ is a white noise error or shock. Note that the double-logarithmic specification of the model is designed to allow for diminishing marginal effects to each continuous determinant of health on life expectancy at birth for female. For example, the elasticity of output $\mathrm{y}$ and with respect to input $\mathrm{x}$ is $=\frac{\partial y}{\partial x} \frac{x}{y}$, where $\frac{\partial y}{\partial x}$ is the marginal effect of $\mathrm{x}$ on $\mathrm{y}$. It can now be seen that if $\frac{x}{y}$ rises (say) by $50 \%$ over the sample period, then the marginal effect of $\mathrm{x}$ on $\mathrm{y}$ must fall proportionately (by 50\%) in order for the elasticity to remain constant.

\section{Estimation Results}

We present the estimation results for our benchmark VEC models in sub-sections 3.1 (qualitative evidence), 3.2 (quantitative evidence) and 3.3 (stability and robustness analysis).

\subsection{Qualitative Evidence}

Table 3 reports the estimation results for each of the six health- indicator VEC models. The left-most column of Table 3 shows the explanatory variables, while each of the remaining six columns shows the estimated VEC model for a health indicator (dependent variable). A few general comments about the overall goodness-of-fit of the models are in order. First, as can be seen from Table 3, the vast majority of the estimated coefficients in the cointegration relationships of the VEC models are statistically significant at the 5 percent level and have the expected signs. Thus, the determinants of health we have selected based on the discussion in section 2 of the paper do, in fact, help increase the average life expectancy for both men and women; and also lower premature deaths and infant mortality in the long run.

Second, the reported $\mathrm{R}^{2}$ metric and the associated Durbin-Watson (DW) statistic suggest that the overall explanatory power of each benchmark model is quite reasonable. It is known since Granger and Newbold (1974) that 'spurious correlation' among trending variables shows up in the form of very high R2, but in such cases the DW statistic approaches a value of zero. As the evidence in table 3 shows, this is clearly not true. Thus the reported $\mathrm{R}^{2}$ value, which ranges from 27.5 percent for the infant mortality model to 57.5 percent for the life expectancy at birth for men model, is highly credible. It is also noteworthy that the residuals from the benchmark models at higher lags are also uncorrelated. In particular, the reported high p-values reject the null hypothesis that residuals that are 5 periods (or16 periods) apart are uncorrelated. This too provides evidence in support of the VEC models.

Third, the 'speed of adjustment' coefficient $\alpha$ has the expected negative sign for all models, except for the LE65M model. This means that any displacements from the long run equilibrium relationships are eventually eliminated. This adjust occurs only partly through changes in the health indicators themselves. For example, the fastest adjustment rate of 18 percent of the previous year's displacement occurs for infant mortality (IM); for most of the other health indicators the adjustment rate is very slow. An important part of the adjustments to equilibrium occurs through changes in the determinants of health. For example, in the cases of life expectancy at birth for female (LE0F), at age 65 for female (LE65F) and for infant mortality (IM), the adjustment to equilibrium occurs through changes in the real health care expenditure per capita.

We now consider the health effects of the two key government policy variables of primary interest to us: government health care spending, LN(HE), (row 1, Table 3) and of social services spending, LN(SS), (row 2, Table 3). Two aspects of these estimates are noteworthy. First, the sign and statistical significance indicate that both of these policy variables have important beneficial effects on population health in the long run. However, government social services spending have beneficial effects on a larger number of health indicators than does spending on health care. For example, LN(SS) significantly increases life expectancy for both men and women and also decreases infant mortality in the long run. By comparison, government spending on health care only increases life expectancy at birth for men and of women at age 65. Second, social services' spending has 
quantitatively larger marginal health benefits for a greater number of health indicators (LN(LE0F), LN(LE0M), LN(LE65M) and LN(IM)) compared to expenditure of health care (LN(LE65F), LN(PD)). Notably, this evidence of the relatively larger marginal health effects of social services spending is consistent with the finding of Dutton et al. (2018).

Table 3. Benchmark vector error correction (VEC) models estimation results

\begin{tabular}{|c|c|c|c|c|c|c|}
\hline \multirow{2}{*}{$\begin{array}{c}\text { HEALTH } \\
\text { DETERMINANTS }\end{array}$} & \multicolumn{6}{|c|}{ HEALTH INDICATORS } \\
\hline & $\Delta \mathrm{LN}(\mathrm{LE} 0 \mathrm{~F})$ & $\Delta \mathrm{LN}(\mathrm{LE} 0 \mathrm{M})$ & $\Delta \mathrm{LN}(\mathrm{LE} 65 \mathrm{~F})$ & $\Delta \mathrm{LN}(\mathrm{LE} 65 \mathrm{M})$ & $\Delta \mathrm{LN}(\mathrm{PD})$ & $\Delta \mathrm{LN}(\mathrm{IM})$ \\
\hline \multirow[t]{2}{*}{$\mathrm{LN}(\mathrm{HE})_{\mathrm{t}-1}$} & 0.0061 & $0.0288^{*}$ & $0.4837 *$ & 0.0396 & -0.1118 & 0.1131 \\
\hline & {$[0.271]$} & {$[2.365]$} & {$[2.589]$} & {$[0.217]$} & {$[-0.840]$} & {$[0.464]$} \\
\hline \multirow[t]{2}{*}{$\mathrm{LN}(\mathrm{SS})_{\mathrm{t}-1}$} & $0.0709 *$ & $0.0325^{*}$ & 0.1622 & $0.3487 *$ & -0.0673 & $-0.4045^{*}$ \\
\hline & [3.104] & [6.501] & {$[1.074]$} & {$[3.113]$} & {$[-0.837]$} & {$[-2.744]$} \\
\hline \multirow[t]{2}{*}{$\mathrm{LN}(\mathrm{GDI})_{\mathrm{t}-1}$} & & $0.1293^{*}$ & $0.8307^{*}$ & & $-0.2273^{*}$ & $-1.7521^{*}$ \\
\hline & & {$[7.680]$} & {$[6.732]$} & & {$[-1.627]$} & {$[-6.485]$} \\
\hline \multirow[t]{2}{*}{$\mathrm{LN}(\mathrm{TOB})_{\mathrm{t}-1}$} & $0.0861 *$ & & $-0.3796^{*}$ & $--0.4131^{*}$ & $0.2232 *$ & $0.2117 *$ \\
\hline & {$[3.152]$} & & {$[-4.147]$} & {$[-3.113]$} & {$[3.623]$} & {$[1.840]$} \\
\hline \multirow[t]{2}{*}{$\mathrm{LN}(\mathrm{ALC})_{\mathrm{t}-1}$} & & & -0.6112 & -0.1192 & 0.0078 & $0.5363 *$ \\
\hline & & & {$[-0.077]$} & {$[-0.971]$} & {$[0.076]$} & {$[2.922]$} \\
\hline \multirow[t]{2}{*}{$\mathrm{LN}(\mathrm{PR})_{\mathrm{t}-1}$} & $-0.0543^{*}$ & $-0.0179 *$ & & & & \\
\hline & {$[-2.727]$} & [ - 1.599$]$ & & & & \\
\hline \multirow[t]{2}{*}{$\mathrm{LN}(\mathrm{SEX})_{\mathrm{t}-1}$} & & & & -73.237 & & \\
\hline & & & & {$[-8.835]$} & & \\
\hline \multirow[t]{5}{*}{ SPEED OF ADJUSTMENT } & -0.0001 & $-0.1228^{*}$ & -0.0075 & $0.0424 *$ & $-0.1414 *$ & -0.1827 \\
\hline & {$[-0.003]$} & $-2.519]$ & {$[-0.417]$} & [2.169] & {$[-1.581]$} & {$[-1.336]$} \\
\hline & $\underline{\mathrm{LN}(\mathrm{HE})}$ & $\Delta \mathrm{LN}(\mathrm{SS})$ & $\Delta \mathrm{LN}(\mathrm{HE})$ & $\Delta \mathrm{LN}(\mathrm{TOB})$ & $\Delta \mathrm{LN}(\mathrm{ALC})$ & $\Delta \mathrm{LN}(\mathrm{HE})$ \\
\hline & $-2.3320^{*}$ & $-2.3320^{*}$ & $-0.3914 *$ & $-0.3451^{*}$ & $-0.6391^{*}$ & $-1.9532 *$ \\
\hline & {$[-3.789]$} & {$[-3.789]$} & {$[-4.199]$} & {$[-2.081]$} & {$[-2.919]$} & {$[-1.953]$} \\
\hline \multirow[t]{2}{*}{$\Delta \mathrm{LN}(\mathrm{HE})_{\mathrm{t}-1}$} & & $-0.0176^{*}$ & & & & -0.5168 \\
\hline & & {$[-1.621]$} & & & & {$[-1.107]$} \\
\hline \multirow[t]{2}{*}{$\Delta \mathrm{LN}(\mathrm{SS})_{\mathrm{t}-1}$} & & $-0.0245^{*}$ & $0.0642 *$ & & $-0.5556^{*}$ & \\
\hline & & {$[-4.004]$} & {$[1.734]$} & & {$[-3.664]$} & \\
\hline \multirow[t]{2}{*}{$\Delta \mathrm{LN}(\mathrm{GDI})_{\mathrm{t}-1}$} & & & $-0.0296 *$ & & $0.2615^{*}$ & \\
\hline & & & {$[-1.774]$} & & [3.456] & \\
\hline \multirow[t]{2}{*}{$\Delta \mathrm{LN}(\mathrm{TOB})_{\mathrm{t}-1}$} & & & & $-3452^{*}$ & & \\
\hline & & & & {$[-2.081]$} & & \\
\hline \multirow[t]{2}{*}{$\Delta \mathrm{LN}(\mathrm{ALC})_{\mathrm{t}-1}$} & $-0.0115^{*}$ & & & & $0.4927^{*}$ & \\
\hline & {$[-2.339]$} & & & & [3.336] & \\
\hline $\mathrm{R}^{2}$ & 0.389 & 0.576 & 0.476 & 0.404 & 0.449 & 0.275 \\
\hline DW statistic & 1.94 & 1.92 & 1.89 & 1.95 & 2.00 & 2.06 \\
\hline Lag $5 \& 16$ (p-value) & $0.55 ; 0.82$ & $0.34 ; 0.52$ & $0.24 ; 0.53$ & $0.66 ; 0.57$ & $0.76 ; 0.90$ & $0.24 ; 0.53$ \\
\hline
\end{tabular}

Note. * indicates statistical significance at the 5 percent level.

Our final comment concerns the health effects of the other determinants of health; especially those of the socio-economic and the life-style determinants of health. The evidence shows that expenditures on tobacco and alcohol consumption have significant detrimental health effects in the long run, including decreasing life expectancy of both male and female and also increasing mortality. Also, the level of household income (LN(GDI)) increases life expectancy and decreases mortality in the long run; while income distribution, measured by the poverty rate (LN(PR)), markedly lowers life expectancy at birth. 


\subsection{Quantitative Evidence}

Statistical significance, which has been the focus of our analysis so far, suffers from an important shortcoming: it may not reflect the true relative health effects of government spending on health and social services over time. Thus, in Table 4, we present further evidence of their quantitative importance in the short, medium and long run. We measure a health input's quantitative importance by the ability of the shocks associated with it to account for the volatility of the health indicators. Volatility of a health indicator is defined as the difference between the actual value and its forecast constructed $h$ periods in the past using the estimated VEC equations (1). We have decomposed the forecast error variance of each health indicator into proportions that are accounted for by each source of disturbance at the forecast horizons $\mathrm{h}=1$ (short run), $\mathrm{h}=5$ (medium run) and $\mathrm{h}=20$ (long run).

For each $\mathrm{h}(=1,5,20)$ and each health indicator, Table 4 reports the variance decomposition results for two alternative causal ordering of the variables in the VEC model. The top row denotes results for ordering 1 in which the health indicator is placed first in the ordering; this ordering does not allow the determinants of health to have an instantaneous effect on the health indicators. The highlighted bottom row denotes the results for ordering 2 in which the health indicator is placed last in the causal ordering; this ordering allows the determinants of health to have an instantaneous effect on the health indicators.

Consider first the short run $(h=1)$ responses of the health indicators under the two causal orderings. Not surprisingly, under ordering $1,100 \%$ of each health indicator's volatility is explained by its own shocks; none by shocks to the determinants of health. But under ordering 2, shocks associated with health care spending emerge as a major contributor to population health. Such shocks account for $17 \%$ of the volatility for LN(LE0F); $22 \%$ of the volatility for $\mathrm{LN}(\mathrm{LE} 0 \mathrm{M}) ; 42 \%$ of the volatility for $\mathrm{LN}(\mathrm{PM})$; while shocks to social services spending play a relatively smaller role; such shocks account for $21 \%$ of the volatility for LN(LE0M) only. At this horizon, shocks to tobacco consumption account for $24 \%$ of the volatility for $\mathrm{LN}(\mathrm{IM})$.

Interestingly, in the medium and the long run, the relative importance of health care and social services spending is completely reversed. For example, in the medium run $(h=5)$, social services spending emerges as the most influential for population health, while spending on health care continue to play an important but diminished role. In fact, a distinct pattern emerges: shocks to $\mathrm{LN}(\mathrm{SS})$ are the prime cause for variability in life expectancy for both women and men; while shocks to $\mathrm{LN}(\mathrm{HE})$ are most important for reducing premature deaths and infant mortality. For example, at $\mathrm{h}=5$, under orderings 1 and 2 respectively, shocks to social services spending account for $19 \%$ and $20 \%$ of $\mathrm{LN}(\mathrm{LE} 0 \mathrm{~F})$ 's volatility; $11 \%$ to $50 \%$ of $\mathrm{LN}(\mathrm{LE} 0 \mathrm{M})$ 's volatility; $24 \%$ to $38 \%$ of $\mathrm{LN}(\mathrm{LE} 65 \mathrm{M})$ 's volatility and $8 \%$ to $32 \%$ of LN(LE65F)'s volatility; while shocks to health care spending account for $5 \%$ to $51 \%$ of $\mathrm{LN}(\mathrm{PM})$ 's volatility and $5 \%$ to $33 \%$ of $\mathrm{LN}(\mathrm{IM})$ 's volatility respectively. This same pattern persists with even stronger force in the long run $(h=20)$. Again, social services spending are the most influential for life expectancy at birth for both male and female, while spending on health care are more important for reducing premature deaths and infant mortality

Table 4. Proportion (\%) of forecast error variance h-years ahead explained by each source of disturbance

\begin{tabular}{|c|c|c|c|c|c|c|c|c|c|}
\hline \multicolumn{10}{|c|}{ Alternative sources of shocks or disturbances } \\
\hline & $\mathrm{h}$ & $\varepsilon_{\mathrm{HE}}$ & $\varepsilon_{S S}$ & $\varepsilon_{\mathrm{GDI}}$ & $\varepsilon_{\mathrm{TOB}}$ & $\varepsilon_{\mathrm{ALC}}$ & $\varepsilon_{\mathrm{PR}}$ & $\varepsilon_{\mathrm{SEX}}$ & $\varepsilon_{\text {OWN }}$ \\
\hline \multirow[t]{6}{*}{ LN(LE0F) } & 1 & 0.0 & 0.0 & & & 0.0 & 0.0 & & 100.0 \\
\hline & & 17.0 & 0.0 & & & 5.0 & 1.0 & & 77.0 \\
\hline & 5 & 0.3 & 19.1 & & & 0.7 & 6.9 & & 73.0 \\
\hline & & 13.0 & 20.0 & & & 6.0 & 4.0 & & 57.0 \\
\hline & 20 & 8.0 & 42.6 & & & 5.0 & 1.0 & & 43.4 \\
\hline & & 3.0 & 44.0 & & & 7.0 & 8.0 & & 38.0 \\
\hline \multirow[t]{6}{*}{ LN(LE0M) } & 1 & 0.0 & 0.0 & & & & 0.0 & & 100.0 \\
\hline & & 22.0 & 21.0 & 9.0 & & & $\mathbf{1 7 . 0}$ & & 30.0 \\
\hline & 5 & 0.0 & 11.0 & 4.0 & & & 1.0 & & 84.0 \\
\hline & & 16.0 & 50.0 & 16.0 & & & 5.0 & & 13.0 \\
\hline & 20 & 0.0 & 8.0 & 7.0 & & & 1.0 & & 84.0 \\
\hline & & 18.0 & 45.0 & 23.0 & & & 5.0 & & 10.0 \\
\hline \multirow[t]{6}{*}{ LN(LE65F) } & 1 & 0.0 & 0.0 & 0.0 & 0.0 & 0.0 & & & 100.0 \\
\hline & & 24.0 & 4.0 & 4.0 & 0.0 & 2.0 & & & 66.0 \\
\hline & 5 & 1.0 & 25.0 & 1.0 & 2.0 & 0.0 & & & 71.0 \\
\hline & & 21.0 & 39.0 & 3.0 & 6.0 & 0.0 & & & 31.0 \\
\hline & 20 & 1.0 & 48.0 & 2.0 & 2.0 & 0.0 & & & 47.0 \\
\hline & & 16.0 & 63.0 & 5.0 & 4.0 & 0.0 & & & 12.0 \\
\hline
\end{tabular}




\begin{tabular}{|c|c|c|c|c|c|c|c|c|}
\hline \multirow[t]{6}{*}{ LN(LE65M) } & 1 & 0.0 & 0.0 & & 0.0 & 0.0 & 0.0 & 100.0 \\
\hline & & 0.0 & 2.0 & & 8.0 & 0.0 & 21.0 & 69.0 \\
\hline & 5 & 1.0 & 8.0 & & 2.0 & 1.0 & 1.0 & 91.0 \\
\hline & & 6.0 & 32.0 & & 7.0 & 2.0 & 19.0 & 34.0 \\
\hline & 20 & 2.0 & 52.0 & & 7.0 & 3.0 & 1.0 & 35.0 \\
\hline & & 1.0 & 58.0 & & 16.0 & 3.0 & 10.0 & 12.0 \\
\hline \multirow[t]{6}{*}{$\mathrm{LN}(\mathrm{PD})$} & 1 & 0.0 & 0.0 & 0.0 & 0.0 & 0.0 & & 100.0 \\
\hline & & 42.0 & 6.0 & 0.0 & 0.0 & 5.0 & & 47.0 \\
\hline & 5 & 5.0 & 6.0 & 14.0 & 1.0 & 0.0 & & 74.0 \\
\hline & & 51.0 & 16.0 & 13.0 & 0.0 & 0.0 & & 20.0 \\
\hline & 20 & 4.0 & 10.0 & 13.0 & 2.0 & 0.0 & & 71.0 \\
\hline & & 48.0 & 22.0 & 12.0 & 0.0 & 0.0 & & 18.0 \\
\hline \multirow[t]{6}{*}{ LN(IM) } & 1 & 0.0 & 0.0 & 0.0 & 0.0 & 0.0 & & 100.0 \\
\hline & & 17.0 & 2.0 & 1.0 & 24.0 & 2.0 & & 54.0 \\
\hline & 5 & 5.0 & 1.0 & 3.0 & 1.0 & 2.0 & & 88.0 \\
\hline & & 33.0 & 2.0 & 3.0 & 20.0 & 6.0 & & 37.0 \\
\hline & 20 & 7.0 & 5.0 & 1.0 & 7.0 & 4.0 & & 76.0 \\
\hline & & 35.0 & 9.0 & 2.0 & 33.0 & 8.0 & & 13.0 \\
\hline
\end{tabular}

Note. At each forecast horizon $\mathrm{h}=1,5,20$ years, the top row shows variance decomposition for causal ordering of variables with the health indicator placed first in the ordering and the bottom (highlighted) row for ordering that places the health indicator last in the ordering.

\subsection{Stability and Robustness Results}

In section 3.1 above, we reported residual properties as evidence of adequacy of the estimated VEC models. This section offers further evidence on model stability and the robustness of our main findings. Model stability requires that the estimated residuals should be mean and variance stationary, while robustness relates to sensitivity of our findings to specification changes, as explained below.

First, we examine whether residuals are mean and variance stationarity by applying the Brown et al. (1975) CUSUM and CUSUMSQ tests respectively. The CUSUM (cumulative sum) test is based on the test statistic $W_{t}=\sum_{r=k+1}^{t} \frac{w_{r}}{s}$, for $\mathrm{t}=\mathrm{k}+1 \ldots . \mathrm{T}$, where $\mathrm{w}_{\mathrm{r}}$ are recursive residuals (one-step ahead forecast errors) and $\mathrm{s}$ is the standard deviation of the recursive residuals. If the parameters of the model remain stable from period to period, then $\mathrm{E}\left(\mathrm{W}_{\mathrm{t}}\right)=0$, if not, then $\mathrm{W}_{\mathrm{t}}$ will tend to diverge from the zero mean value line. The statistical significance of divergence from the zero mean line is assessed by reference to a pair of 5\% significance lines, as shown Figures 1a to 6a below. Movement of $\mathrm{W}_{\mathrm{t}}$ outside of the critical lines indicates mean non-stationary. Clearly none of six VEC models suffers from mean non-stationarity. Similarly, the CUSUMSQ test is based on the cumulative sum of the squares of the recursive residuals. The results of this test are shown in Figures $1 \mathrm{~b}$ to $6 \mathrm{~b}$. Again, the figures show little or no evidence that the VEC models suffer from variance non-stationary. Overall, the results of the residuals analysis suggest that the VEC models have successfully accounted for both trend and variance non-stationary problems in our observational health data.

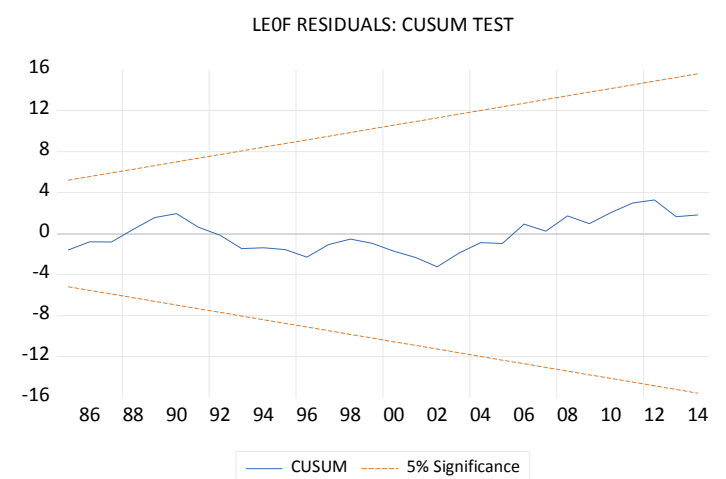

Figure 1a

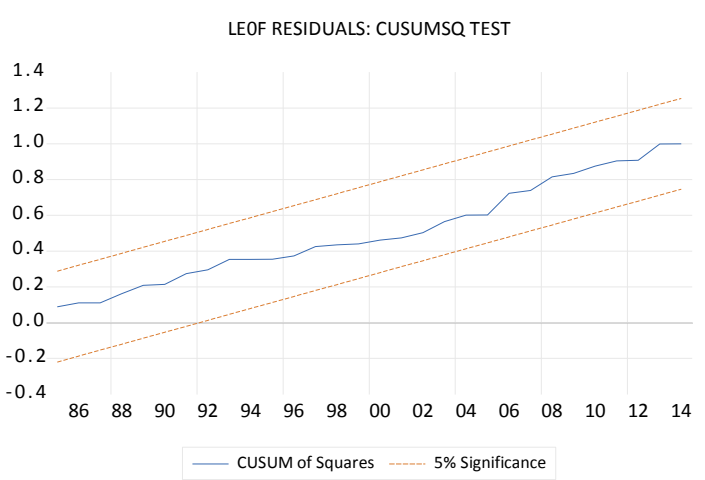

Figure 1b 


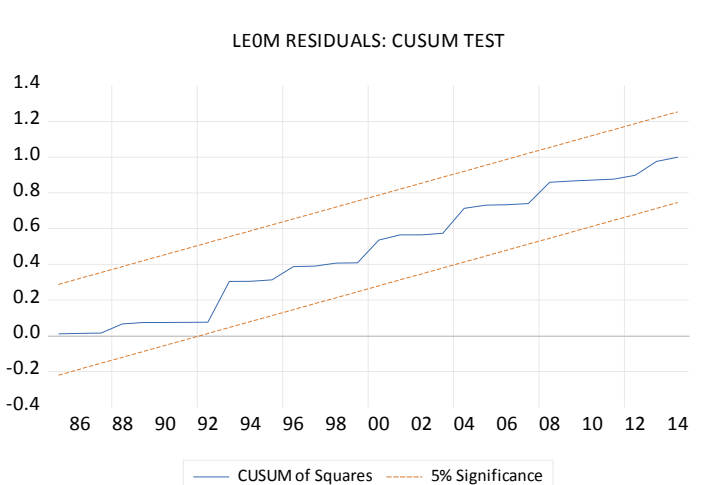

Figure 2a

LE65F RESIDUALS: CUSUM TEST

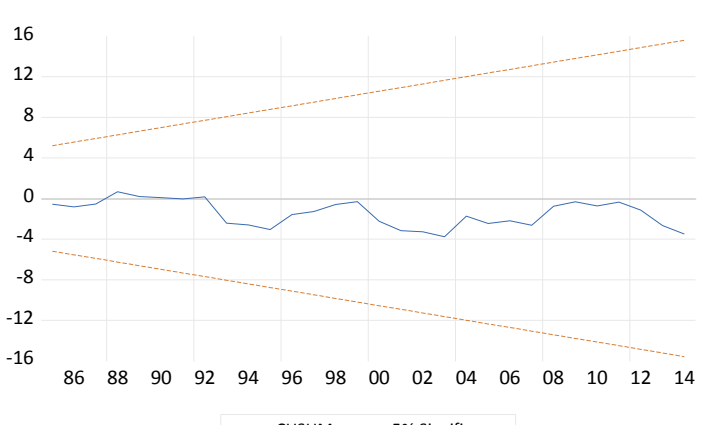

Figure $3 \mathrm{a}$

LE65M RESIDUALS: CUSUM TEST

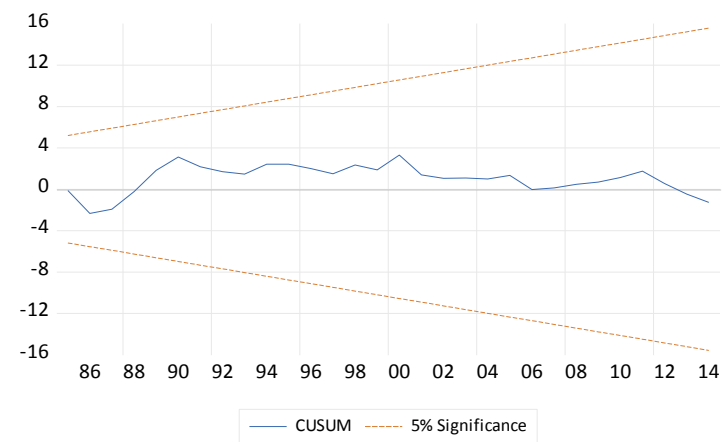

Figure $4 \mathrm{a}$

IM RESIDUALS: CUSUM TEST

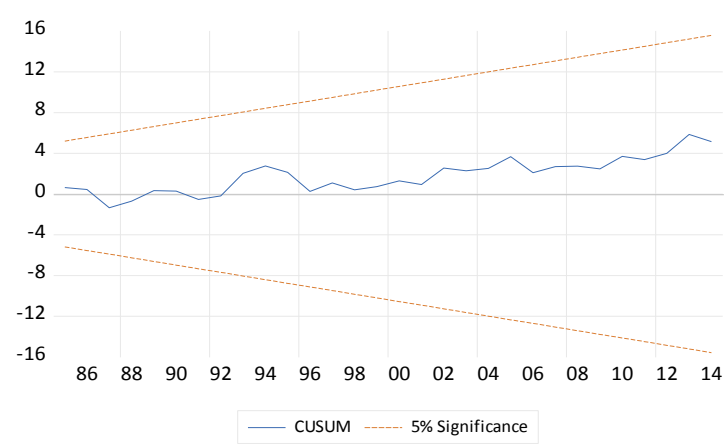

Figure 5a
LEOM Residuals: CUSUMSQ TEST

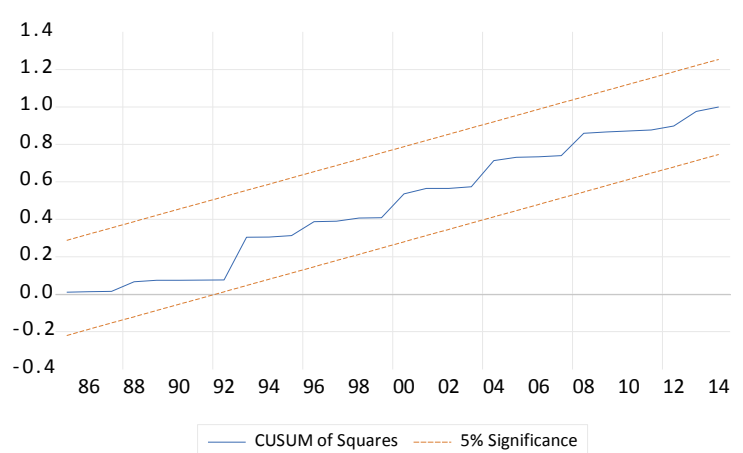

Figure $2 b$

LE65F: CUSUMSQ TEST

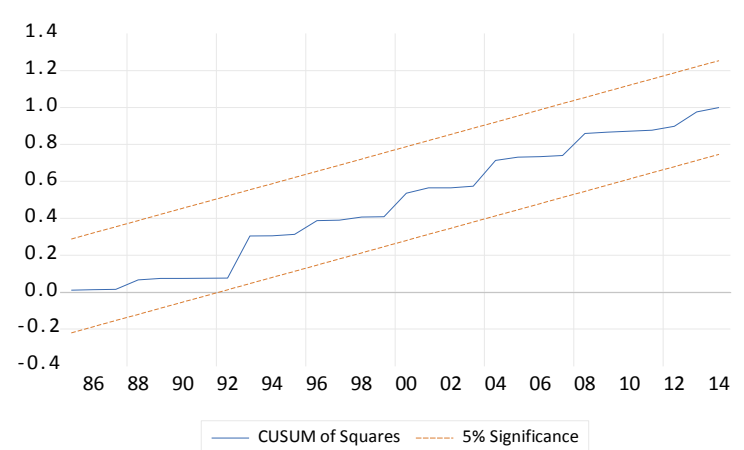

Figure $3 b$

LE65M RESIDUALS: CUSUMSQ TEST

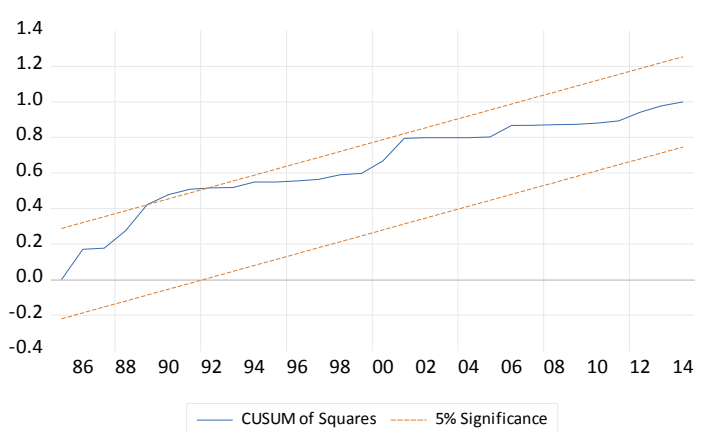

Figure $4 b$

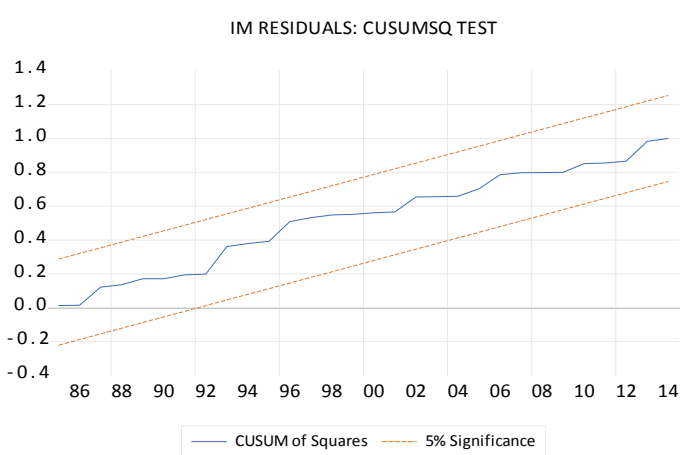

Figure 5b 


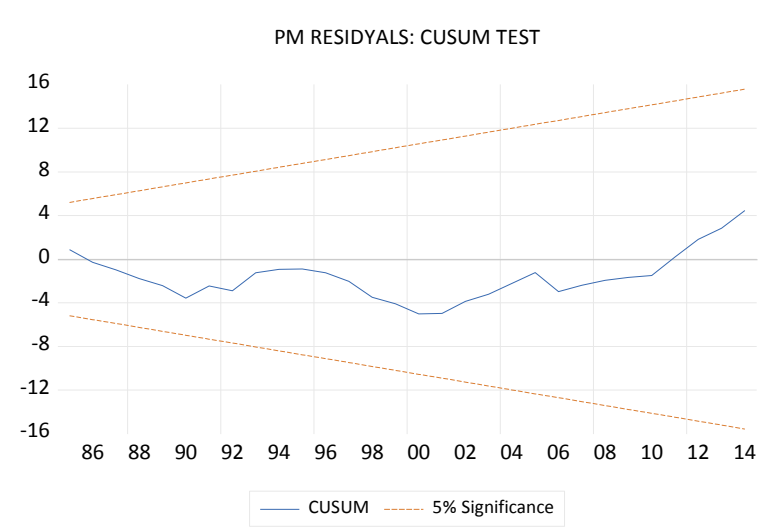

Figure 6a

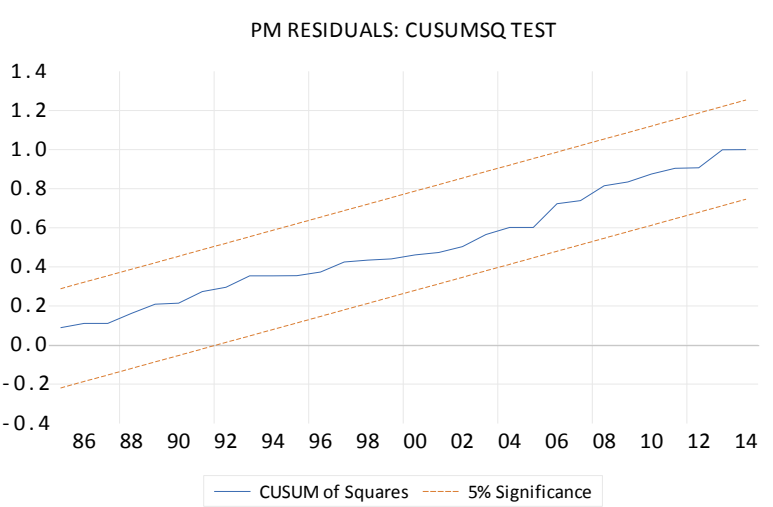

Figure 6b

Finally, for robustness check, we examine the sensitivity of our benchmark results (presented in Table 4) to two types of specification changes: (1) adding an exogenous explanatory variable to the benchmark VEC models and (2) allowing longer (five year) lags to account for possible cumulative effects of smoking and drinking on health outcomes. The added exogenous variables are selected from our list of the ten determinants of health, with the proviso that the variables are not cointegrated with the health indicators (and therefore, already included as endogenous variables in the models). To save space, we do not report the details of these robustness checks in the paper; instead, here we only summarize the major changes that occur to the variance decomposition results reported in Table 4 under the two causal orderings.

Both the addition of an exogenous variable and allowing for longer lags, alter the variance decomposition results in table 4 in two ways across the models. First, in both cases, the percentage of each health indicator's volatility (forecast error variance) explained by the model's explanatory variables increases marginally. Second, across all models the proportions of each health indicator's explained volatility accounted for by the social services spending and the health care spending changes (compared to Table 4); but in no case, the resulting changes are large enough to alter the relative importance of health care vs. social services spending. Thus, the benchmark findings reported in table 4 are fairly robust to both static and dynamic specification changes.

\section{Conclusions and Limitations}

In this paper we have investigated the short and long run health effects government spending on health care vs. social services across six different population health indicators. Based on both statistical significance and (quantitative) variance decomposition analysis, we have reached two substantive conclusions.

First, both government health care and social services spending have major health benefits in terms of increasing life expectancy of both male and female and decreasing mortality. These two public-sector determinants of health matter more than the socio-economic, demographic and the life-style determinants of population health. Second, in the short run, the quantitative health benefits of government spending on health care are systematically bigger than the effects of spending on social services. This pattern is reversed in the medium and the long run. This is because while the health benefits of health care spending diminish over time; the benefits of social services spending increase markedly so that they become quantitatively bigger than those of health care spending.

The policy implication of the findings of this paper differs markedly from that derived in existing studies. While existing studies typically recommend a reallocation of government budget more towards social services and away from health care, the findings of this paper suggest a trade-off between the short and long run health effects of government spending on health care and social services. Any re-allocative policy must take this trade-off into account.

We conclude this paper by underlining two limitations of the study. First, even though we have examined the health effects of the major determinants of health that are typically studied in the literature, we have excluded such variables as nutritional intake and human genetics from this study due to the lack of time series data. To the extent that these excluded variables have independent health effects beyond the effects of the included variables, the marginal health effects of the included variables may be biased. Thus, the results reported in table 3 and table 4 should be viewed as ballpark estimates. Second, since both the delivery and most of the financing of health services are provincial responsibilities in Canada, the findings of this paper may not apply to each individual 
province in the country. This is especially true with regard to any policy of a reallocation of resources from social services to health care. A province by province study is needed to determine where the results based on aggregate data holds and where it does not. This is an issue for future studies.

\section{Acknowledgements}

This research was supported by an internal grant from the Laurentian University Research Fund under grant number LURF Award W19 (41-1-6090723).

\section{References}

Banerjee, A., Dolado, J., Galbraith, J. W., \& Hendry, D. F. (1993). Co-Integration, Error-Correction, and the Econometric Analysis of Non-Stationary Data (pp. 70-81). Oxford University Press. https://doi.org/10.1093/0198288107.001.0001

Bradley, E. H., \& Taylor, L. A. (2013). The American health care paradox: Why spending more is getting us less. New York: Public Affairs.

Bradley, E. H., Canavan, M., Rogan, E., et al. (2016). Variation in health outcomes: the role of spending on social services, public health, and health care, 2000-09. Health Affairs (Millwood), 35, 760-8. https://doi.org/10.1377/hlthaff.2015.0814

Bradley, E. H., Elkins, B. R., Herrin, J., \& Elbel, B. (2011). Health and social services expenditures: associations with health outcomes. BMJ Quality Safety, 1-6. https://doi.org/10.1136/bmjqs.2010.048363

Brown, R. L., Durbin, J., \& Evans, J. M. (1975). Techniques for Testing the Constancy of Regression Relationships over Time. Journal of the Royal Statistical Society, Series B, 35, 149-192. https://doi.org/10.1111/j.2517-6161.1975.tb01532

Crémieux, P. Y., Ouellette, P., \& Pilon, C. (1999). Health care spending as determinants of health outcomes. Health Economics, 627-639. https://doi.org/10.1002/(SICI)1099-1050(199911)8:7<627::AID-HEC474>3.0.CO;2-8

Dahlgren, G., \& Whitehead, M. (1991). Policies and Strategies to Promote Social Equity in Health. Stockholm, Sweden: Institute for Futures Studies.

Dutton, J. D., Forest, P. G., Kneebone, R. D., \& Zwicker, J. D. (2018). Effects of provincial spending on social services and health care on health outcomes in Canada: An observational longitudinal study. CMAJ, 190, E66-71. https://doi.org/10.1503/cmaj.170132

Grossman, M. (1972). The Demand for Health: A Theoretical and Empirical Investigation. Number 119 in Occasional Paper. National Bureau of Economic Research, New York.

Hall, R. E., \& Jones, C. I. (2007). The Value of Life and the Rise in Health Spending. The Quarterly Journal of Economics, 122(1), 39-72. https://doi.org/10.1162/qjec.122.1.39

Hitiris, T., \& Posnett, J. (1992). The determinants and effects of health expenditure in developed countries. Journal of Health Economics, 11(2), 173-81. https://doi.org/10.1016/0167-6296(92)90033-W

Johanssen, S. (1988). Statistical analysis of cointegrated vectors. Journal of Econometric Dynamics and Control, 12, 231-54. https://doi.org/10.1016/0165-1889(88)90041-3

Kneebone, R., \& Wilkins, M. (2016). Canadian provincial government budget data, 1980/81 to 2013/14. Canadian Public Policy, 42, 1-19. https://doi.org/10.3138/cpp.2015-046

Kwiatkowski, D., Phillips, P. C. B., Schmidt, P., \& Shin, Y. (1992). Testing the null hypothesis of stationarity against the alternative of a unit root. Journal of Econometrics, 54, 159-178. https://doi.org/10.1016/0304-4076(92)90104-Y

Liaropoulos, L., \& Goranitis, I. (2015). Health care financing and the sustainability of health systems. International Journal for Equity in Health, 14(1). https://doi.org/10.1186/s12939-015-0208-5.

McDaid, D., Sassi, F., \& Merkur, S. (2015). Promoting Health, Preventing Disease: The economic case. McGraw Hill (WHO).

Phillips, D. (1993). Urbanization and human health. Parasitology, 106(S1), S93-S107. https://doi.org/10.1017/S0031182000086145.

Pickett, K. E., \& Wilkinson, R. G. (2015). Income inequality and health: A causal review. Social Science and Medicine, 128, 316-26. https://doi.org/10.1016/j.socscimed.2014.12.031. 
Rose, G., Khaw, K. T., \& Marmot, M. (2008). Rose's strategy of preventive medicine. Oxford (UK): Oxford University Press. https://doi.org/10.1093/acprof:oso/9780192630971.001.0001

Skinner, B. J., \& Mark, R. (2011). Canada's Medicare Bubble: Is government spending sustainable without user-based funding? Studies in Healthcare Policy. Retrieved from http://www.fraserinstitute.org

Wilkinson, R., \& Pickett, K. (2011). The Spirit Level: Why Greater Equality Makes Societies Stronger. New York: Bloomberg Press.

World Health Organization. (1991). Health and the cities: A global overview. Technical discussion on strategies for health for all in the face of rapid urbanization. A44/Technical discussion/2. Geneva: WHO.

World Health Organization. (2001). Encouraging people to stop smoking. Department of mental health and substance dependence, World health organization, Geneva, Switzerland. Retrieved from https://www.who.int/mental_health/evidence/stop_smoking_whomsdmdp01_4.pdf

\section{Notes}

Note 1. We use the low income measure (LIM) to assess how people fare compared with the general population. Statistics Canada estimates the poverty line as 50 per cent of the national median income. The poverty rate is calculated as the share of the population with disposable incomes (after taxes and government transfers) below this poverty line.

Note 2. There is some evidence that the series LN(LE65F) may be integrated of order 2. To avoid differencing of variables and loss of long run information, we have added a time trend in the (VEC) model for this series (see section 2.5).

\section{Appendix}

This appendix describes each variable and provides the source of raw data for each series.

\begin{tabular}{ll}
\hline Variable Description & Data Source \\
\hline Life expectancy at birth for male (years) & Statistics Canada Table: 13-10-0114-01 \\
Life expectancy at birth for female (years) & Statistics Canada Table: 13-10-0114-01 \\
Life expectancy at age 65 for male (years) & Statistics Canada Table: 13-10-0114-01 \\
Life expectancy at age 65 for female (years) & Statistics Canada Table : 13-10-0114-01 \\
Premature death rate (per100, 000 population) & Statistics Canada Table: 13-10-0744-01 \\
Infant mortality rate (per 1000) & Statistics Canada Table: 13-10-0368-01 \\
Public-Health expenditure per capita current dollars & CIHI Tables D.1.1.3 - D.1.10.3 \\
Social services expenditure (millions of current dollars) & Data Appendix, Kneebone and Wilkins, 2016* \\
Real household gross domestic income per capita, 2002 dollars Statistics Canada Table: 36-10-0229-01 \\
Population 65+ (Percentage) & Statistics Canada Table: 1710-0005-01 \\
Unemployment rate (\%) & Statistics Canada Table 14-10-0023-01 \\
Real household expenditure on tobacco products & Statistics Canada Table: 36-10-0225-01 \\
Real household expenditure on Alcohol & Statistics Canada Table: 36-10-0225-01 \\
Urbanization Rate (\%) & Statistics Canada Table: 36-10-0229-01 \\
Proportion of Female in the population & Statistics Canada Table: 17-10-0005-01 \\
Poverty Rate (\%) & Statistics Canada file:///E:/Poverty\%20-\%20Provincial.html \\
Consumer price Index, 2002 & Statistics Canada Table 18-10-0005-01 \\
$*$ Kneebone R., Wilkins M. 2016. Canadian provincial government budget data. 1980/81 to 2013/2014. Canadian Public Policy 42: 1-19. \\
\hline
\end{tabular}

\section{Copyrights}

Copyright for this article is retained by the author(s), with first publication rights granted to the journal.

This is an open-access article distributed under the terms and conditions of the Creative Commons Attribution license (http://creativecommons.org/licenses/by/4.0/). 